\title{
An Optimally Diffusive Coarse Mesh Finite Difference Method to Accelerate Neutron Transport Calculations
}

\author{
Ang Zhu*, Michael Jarret, Yunlin Xu, Brendan Kochunas, Edward Larsen, and \\ Thomas Downar
}

\author{
Department of Nuclear Engineering \& Radiological Science \\ University of Michigan, Ann Arbor, 48105, USA \\ *angzhu@umich.edu
}

\begin{abstract}
A new optimally diffusive Coarse Mesh Finite Difference (odCMFD) method is proposed. The new method generalizes the Coarse Mesh Finite Difference (CMFD) and partial current-based CMFD (pCMFD) methods by adding an artificial term to the diffusion coefficient. The standard CMFD and PCMFD methods preserve the net current and partial currents respectively and can greatly reduce the spectral radius for solving neutron transport problems. Linearized Fourier analysis shows that these methods essentially differ only by the definition of the diffusion coefficient - the pCMFD diffusion coefficient contains an additional "artificial" term, usually taken to be $1 / 4$ the coarse mesh size. In this paper, the magnitude of the artificially diffusive term is numerically investigated and optimized using Fourier analysis. The results show that the optimal coefficient increases as the coarse cell optical thickness increases. Also, the optimal value is always smaller than the PCMFD value. This indicates that the pCMFD method overcorrects the diffusion coefficient which increases the spectral radius. A simple polynomial fitting was found for implementing the odCMFD in the MPACT code to perform calculations for realistic problems. Numerical MPACT results for a two-region homogeneous problem, a 2D C5G7 problem, a VERA problem 5 and a 2D BWR Peach Bottom Unit 2 core problem agree well with the Fourier analysis and confirm previous research results that : 1) CMFD converges faster than pCMFD for optically thin coarse grids, but CMFD diverges for optically thick coarse grids; 2) pCMFD is unconditionally stable for all coarse grids; and show that the newly proposed odCMFD is the most efficient among these three methods for all cases. The new method has a similar convergence rate as CMFD for optically thin coarse grids, is faster than both CMFD and pCMFD for coarse grids with intermediate optical thickness, and is unconditionally stable and faster than pCMFD for optically thick coarse grids.
\end{abstract}

Key Words: odCMFD, CMFD, pCMFD, Neutron Transport, Fourier analysis

\section{INTRODUCTION}

Three-dimensional, full-core modeling with pin-resolved detail has become the state of the art for computational simulations of neutron transport for nuclear reactors. However, the computational intensiveness of these problems is challenging, especially for cycle depletion [1] and time-dependent transient analysis [2][3] which requires the solution of multiple steady state eigenvalue/transient fixed source problems.

Extensive studies of various acceleration methods for neutron transport have been performed, and their convergence and stability have been analyzed both theoretically and 
numerically [4][5]. Of these methods, the Coarse Mesh Finite Difference (CMFD) method, which originates from the early 1980's [6] for diffusion problems has gained popularity in recent decades for its simplicity and efficiency in accelerating the steady state [7][8] and transient neutron transport calculations [9][10][11]. However, numerical and theoretical results show that for realistic whole-core calculations, the CMFD method becomes unstable for problems with large coarse mesh cells [12] [13][14][15].

More recently, a variant of the CMFD method, called partial current-based CMFD (pCMFD), was developed by Cho [16] and found to be unconditionally stable for monoenergetic infinite homogeneous medium problems [15][17]. A linearized Fourier analysis [12][18] demonstrated that the pCMFD is theoretically and algebraically "equivalent" to the CMFD method if an additional term $\theta \Delta$ - shown in Eq. (1) - is added to the diffusion coefficient (referred as artificially diffusive CMFD method), where $\Delta$ denotes the coarse mesh size. This extra term effectively suppresses the spectral radius for large coarse mesh sizes, for which the CMFD method can be unstable. However, the Fourier analysis of pCMFD, along with numerical results has shown that pCMFD will be slower than CMFD for intermediate and smaller coarse mesh sizes [17]. This may explain in part why the pCMFD has not been more widely adopted.

$$
D_{p C M F D}=\frac{1}{3 \Sigma_{t r}}+\theta \Delta \quad, \quad \theta_{p C M F D}=\frac{1}{4}
$$

The idea of optimally diffusive CMFD originates from [19], where an "exact" diffusion coefficient is developed for discrete transport solutions in planar geometry with a quadratic source and equal sizes of the fine and coarse meshes. The "exact" diffusion coefficient differs from the standard diffusion coefficient shown in Eq. (1) by adding different coefficients. These are shown in Eq. (2), in which $\mu_{n}$ and $\omega_{n}$ are the 1-D discrete ordinates and weights, and $\alpha_{n}$ is the step characteristics auxiliary equation coefficient defined in Eq.(3).

$$
\begin{gathered}
\theta_{o d}=\frac{1}{4} \sum_{n=1}^{N} \mu_{n} \alpha_{n} \omega_{n}, \\
\alpha_{p}=\frac{1+e^{-\Sigma_{t} \Delta / \mu_{n}}}{1-e^{-\Sigma_{t} \Delta / \mu_{n}}}-\frac{2 \mu_{n}}{\Sigma_{t} \Delta} .
\end{gathered}
$$


The $\theta$ value as a function of optical thickness $\Sigma_{t} \Delta$ based on Eq. (2) is shown in Figure 1. The value of $\theta$ changes dramatically as a function of the coarse cell optical thickness, ranging from the (CMFD) value of 0 for optically thin coarse grids, to the pCMFD value of $1 / 4$ for optically thick coarse grids. The previous work [19] investigated the optimal $\theta$ value from the viewpoint of accuracy, but it is straightforward to demonstrate that the "exact" diffusion solver can efficiently accelerate the discrete transport calculation in planar geometry with a quadratic source and equal sizes of the fine and coarse meshes by one iteration since the accelerator calculates the same result as the high order solver. Furthermore, such an "exact" approximation of the diffusion equation to the transport equation may not be possible for a complicated geometry especially with multiple fines cells per coarse cell. However, this motivates the research here to develop an optimal set of optical thickness dependent $\theta$ rather than directly using 0 for CMFD and $1 / 4$ for pCMFD.

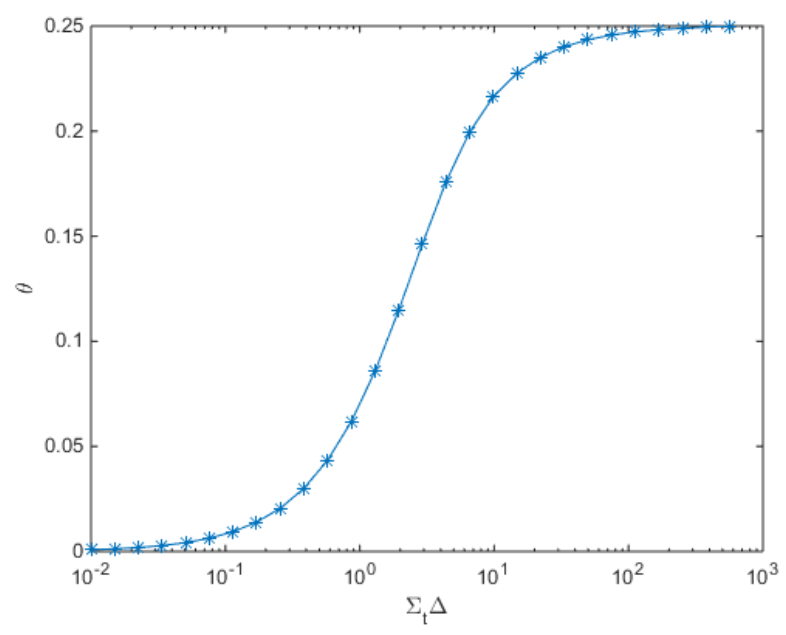

Figure 1: Value of $\theta$ based on Eq. (2).

A similar idea, referred to as generalized coarse-mesh rebalance (GCMR), was proposed by Yamamoto [20], in which a Fourier analysis was used to obtain an optimal multiplication factor that was applied to diffusion coefficients for the coarse mesh rebalance (CMR) and CMFD methods for solving fixed source problems. The GCMR method showed improvement inthe spectral radius over the traditional CMR and CMFD methods for monoenergetic infinite homogeneous problems. However, the analysis stopped at the theoretical analysis, when the author argued that the optimal multiplication factor would be too difficult to be determined for complex geometries. 
Recently, an under-relaxation factor [21] to resolve 2D/1D stability issues was successfully implemented into MPACT (Michigan Parallel Characteristic Transport)[9]. This work demonstrated that "optimized" coefficients derived from the Fourier analysis of the model problem can improve the convergence of complicated complex realistic heterogeneous calculations.

In the work here, an optimally diffusive CMFD (odCMFD) method is proposed and demonstrated in MPACT. The optimal "artificially diffusive" $\theta$ value is investigated which minimizes the spectral radius of the coupled high-order transport and low-order CMFD calculations for the steady state eigenvalue problems of a variety of cases.

The remainder of this paper is organized as follows. Section 2 presents a detailed overview of the steady state neutron transport equation and the various CMFD methods, including: the standard CMFD method, the PCMFD method, and the newly-proposed odCMFD method. Section 3 is devoted to a Fourier analysis to determine the optimal $\theta$ value required for the odCMFD method. Numerical comparisons of the CMFD, PCMFD and the odCMFD methods are also provided. Section 4 presents numerical results for the CMFD method and two of its variants for a two-region homogeneous problem, a 2D C5G7 problem, a VERA problem 5 and a 2D BWR Peach Bottom Unit 2 core problem using the MPACT (Michigan PArallel Charactistics based Transport) code [8]. A summary and final conclusions are given in Section 5 .

\section{CMFD ACCELERATED NEUTRON TRANSPORT METHODS}

\subsection{The Neutron Transport Equation}

We consider the multi-group steady state neutron transport equations shown in Eq. (4), where the solution can be obtained by the Method of Characteristics (MOC), Discrete Ordinates $\left(\mathrm{S}_{\mathrm{N}}\right)$ methods, etc.

$$
\begin{aligned}
\boldsymbol{\Omega} \nabla & \varphi_{g}(\mathbf{r}, \boldsymbol{\Omega})=-\Sigma_{t, g}(\mathbf{r}) \varphi_{g}(\mathbf{r}, \boldsymbol{\Omega}) \\
& +\sum_{g^{\prime}=1}^{G} \int_{0}^{4 \pi} \Sigma_{s, g^{\prime} \rightarrow g}\left(\mathbf{r}, \boldsymbol{\Omega} \boldsymbol{\Omega} \boldsymbol{\Omega}^{\prime}\right) \varphi_{g^{\prime}}\left(\mathbf{r}, \boldsymbol{\Omega}^{\prime}\right) d \Omega^{\prime} \\
& +\frac{1}{4 \pi} \chi_{g}(\mathbf{r}) \frac{1}{k_{\text {eff }}} \sum_{g^{\prime}=1}^{G} v \Sigma_{f, g^{\prime}}(\mathbf{r}) \phi_{g^{\prime}}(\mathbf{r}) .
\end{aligned}
$$

Using standard notation, the terms in this equation are defined as:

$\varphi_{g}$ : group $g$ angular flux; 
$\phi_{g}:$ group g scalar flux;

$\chi_{g}:$ fission spectrum for group $g$;

$\Sigma_{s, g^{\prime} \rightarrow g}$ : scattering cross-section from group $g$ ' to group $g$;

$\Sigma_{t, \mathrm{~g}}:$ total cross-section for group $q$;

$v$ : averaged neutron emitted per fission reaction;

$\Sigma_{f, \mathrm{~g}}$ : fission cross-section for group $g$;

$k_{\text {eff }}$ : steady state eigenvalue.

\subsection{The Coarse Mesh Finite Difference Method}

As discussed above, the iterative solution of the neutron transport equation using a source iteration can be extremely slow, and therefore is often accelerated by CMFD. The CMFD method starts with the neutron balance equation in Eq. (5), where the $m$ denotes the coarse mesh node index, $s$ means a surface of node $m$, and $V_{m}$ and $A_{s}$ are the volume and surface area of node $m$ and surface $s$, respectively.

$$
\sum_{s} A_{s} J_{g, s}^{n e t}+\sum_{r, g, m} \Phi_{g, m} V_{m}=V_{m}\left(\frac{\chi_{g}}{k_{e f f}} \sum_{g^{\prime}=1}^{G} v \Sigma_{f, g^{\prime}, m} \Phi_{g^{\prime}, m}+\sum_{\substack{g^{\prime}=1 \\ g^{\prime} \neq g}}^{G} \Sigma_{s, g g^{\prime}, m} \Phi_{g^{\prime}, m}\right)
$$

To compute the coefficients of Eq. (5) on the coarse grid, the cross sections and flux are homogenized as in Eq. (6), where the superscript $i$ denotes the fine mesh cells in each CMFD coarse cell.

$$
\Sigma_{g, x, m}=\frac{\sum_{i=1}^{I^{m}} \sum_{g, x, m}^{i} \phi_{g, m}^{i} V^{m, i}}{\sum_{i=1}^{I^{m}} \phi_{g, m}^{i} V^{m, i}} \quad, \quad \Phi_{g, m}=\frac{\sum_{i=1}^{I^{m}} \phi_{g, m}^{i} V^{m, i}}{\sum_{i=1}^{I^{m}} V^{m, i}} .
$$


The essential idea of the CMFD method is to preserve the net neutron current at all surfaces by adding a correction term, $\hat{D}$, shown in Eq. (7). In this equation the subscript $m+1 / 2$ denotes the surface between node $m$ and node $m+1$.

$$
J_{g, m+1 / 2}^{n e t}=-\tilde{D}_{g, m+1 / 2}\left(\Phi_{g, m+1}-\Phi_{g, m}\right)+\hat{D}_{g, m+1 / 2}\left(\Phi_{g, m}+\Phi_{g, m+1}\right)
$$

Here the $\tilde{D}$ term is the first-order finite difference coefficient defined in Eq. (8). Not shown are the expressions for $\tilde{D}$ and $\hat{D}$ at the problem boundary which can be derived in a straightforward manner.

$$
\tilde{D}_{g, m+\frac{1}{2}}=\frac{2 D_{g, m+1} D_{g, m m}}{h_{m+1} D_{g, m}+h_{m i} D_{g, m+1}}, \quad D_{g, m}=\frac{1}{3 \Sigma_{i r, g, m}} .
$$

After each higher order transport sweep, the non-linear coupling coefficients, $\hat{D}$, are calculated at each coarse mesh surface using Eq. (9), in which the superscript high denotes the result from the high order transport solution.

$$
\hat{D}_{g, m+1 / 2}=\frac{J_{g, m+1 / 2}^{n e t h i g h}+\tilde{D}_{g, m+1 / 2}\left(\phi_{g, m+1}-\phi_{g, m}\right)}{\left(\phi_{g, m+1}+\phi_{g, m}\right)}
$$

Note that Eq. (9) ensures that the coarse mesh current defined by Eq. (7) is equivalent to the current computed from the transport solution at convergence. The definition from Eq. (7) is then introduced into Eq. (5) and the resulting low-order matrix eigenvalue problem can be solved to obtain the transport eigenvalue, where the CMFD flux and eigenvalue in Eq. (5) are the unknowns.

\subsection{The Partial Current-Based CMFD Method}

Instead of preserving the total net current on each coarse mesh cell face, the pCMFD method preserves the partial currents in the positive and negative directions as shown in Eq. (10), thus requiring two correction coefficients. 


$$
\begin{aligned}
& J_{g, m+1 / 2}^{+}=-\frac{1}{2} \tilde{D}_{g, m+1 / 2}\left(\Phi_{g, m+1}-\Phi_{g, m}\right)+\hat{D}_{g, m+1 / 2}^{+} \Phi_{g, m} \\
& J_{g, m-1 / 2}^{-}=\frac{1}{2} \tilde{D}_{g, m+1 / 2}\left(\Phi_{g, m+1}-\Phi_{g, m}\right)+\hat{D}_{g, m+1 / 2}^{-} \Phi_{g, m+1}
\end{aligned}
$$

The $\hat{D}^{ \pm}$terms in Eq. (10) can be calculated similarly to $\hat{D}$ in Eq. (9) by setting the current on the left hand side of Eq. (10) equal to the value estimated by transport solution :

$$
\begin{aligned}
& \hat{D}_{g, m+1 / 2}^{+}=\frac{2 J_{g, m+1 / 2}^{+, h i g h}+\tilde{D}_{g, m+1 / 2}\left(\phi_{g, m+1}-\phi_{g, m}\right)}{2 \phi_{g, m}}, \\
& \hat{D}_{g, m+1 / 2}^{-}=\frac{2 J_{g, m+1 / 2}^{-, h i g h}-\tilde{D}_{g, m+1 / 2}\left(\phi_{g, m+1}-\phi_{g, m}\right)}{2 \phi_{g, m+1}} .
\end{aligned}
$$

In the pCMFD method the net current in Eq. (5) is can then be expressed as:

$$
J_{g, m+1 / 2}^{n e t}=-\tilde{D}_{g, m+1 / 2}\left(\Phi_{g, m+1}-\Phi_{g, m}\right)-\left(\hat{D}_{g, m+1 / 2}^{-} \Phi_{g, m+1}-\hat{D}_{g, m+1 / 2}^{+} \Phi_{g, m}\right)
$$

Just as in the standard CMFD method, Eq. (12) can be inserted back into Eq. (5) and a low-order matrix eigenvalue problem can be solved where the pCMFD scalar fluxes and eigenvalue are the unknowns.

\subsection{The Optimally Diffusive CMFD Method}

The odCMFD method uses the same equations as the standard CMFD method with only one minor modification. Instead of the conventional definition of the diffusion coefficient in Eq. (8), the diffusion coefficient is modified to include an arbitrary additive component $\theta$ as shown by Eq. (13).

$$
D_{g, m}=\frac{1}{3 \Sigma_{t r, g, m}}+\theta_{o d} \Delta
$$

The optimal $\theta_{\text {od }}$ is determined based on the Fourier analysis method discussed in Section 3. The odCMFD method can be solved using a CMFD or pCMFD code by changing only the diffusion coefficient in Eq. (8) to Eq. (13). 
The odCMFD essentially generalizes the CMFD and pCMFD methods. They differ only by the value of $\theta$ in Eq. (13); the $\theta$ values for these methods can be a problemdependent optimized values (defined below), 0 , or $1 / 4$.

\subsection{CMFD Algorithm in MPACT}

The eigenvalue calculation scheme of MPACT [8] provides an illustration of the coupling between the neutron transport solver and the CMFD acceleration solvers. MPACT is a three-dimensional whole core transport code that is capable of generating sub-pin level power distributions. This is accomplished by obtaining the integral transport solutions to the heterogeneous reactor problem in which the actual pin-resolved geometrical configuration of fuel components such as the pellet and cladding is explicitly retained during the flux solution. The cross section data needed for the neutron transport calculation is obtained directly from a multi-group microscopic cross section library similar to those used in lattice physics codes. Hence, MPACT involves neither a priori homogenization nor group condensation for the core spatial solution. The steady-state integral transport solution is obtained by a means of the method of characteristics (MOC), which employs discrete ray tracing and is accelerated by the CMFD method discussed above.

The CMFD algorithm of the MPACT code is shown in Figure 2. The CMFD and MOC calculations are iteratively performed until converged, where the CMFD method is used to generate the coarse mesh solution for MOC, and the MOC solution provides the estimates of the coupling coefficients $\hat{D}$ for the low-order CMFD calculations. The convergence criteria for CMFD is usually set much tighter than MOC, since the CMFD calculation is much less computationally expensive. 


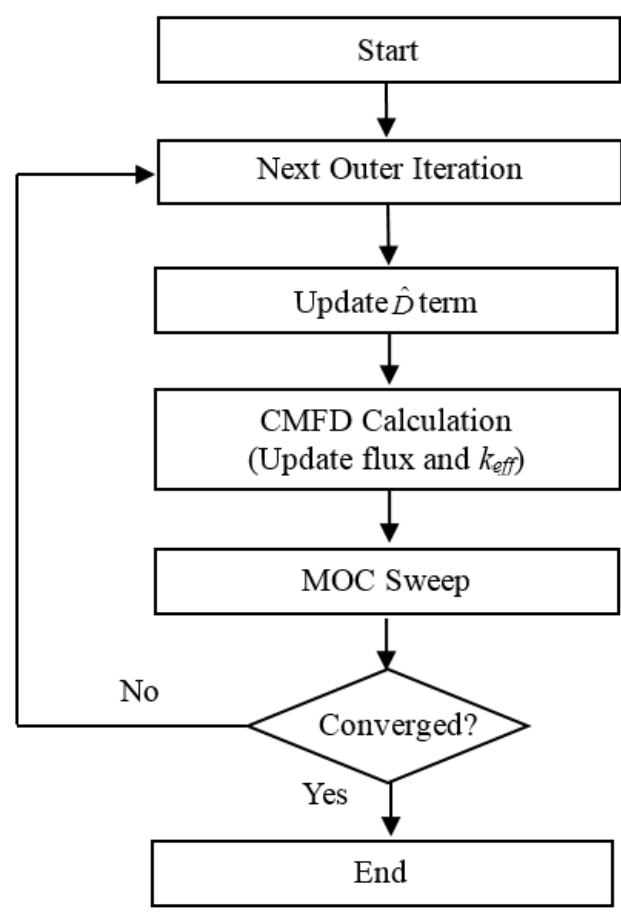

Figure 2: Flowchart of the MPACT code

\section{FOURIER ANAL YSIS OF CMFD}

The Fourier analysis is a standard technique widely used to investigate the asymptotic convergence rate for fixed point iterative methods, and has been used widely in the analysis of CMFD acceleration [12][13][14][15]. The standard procedure includes: 1) definition of a model problem (e.g. a monoenergetic homogeneous infinite media problem); 2) linearization of the equations near the solution; 3) expressing the solution of linearized equations (the error terms) as Fourier modes; and 4) solving for the coefficients of the Fourier modes. These coefficients are the eigenvalues of the iterative method, with the largest being the spectral radius associated with the problem.

The Fourier analysis used in this paper is based on the work in [13] and only a simplified version is provided below. For all the Fourier analysis performed in this paper, an $S_{32}$ Chebyshev-Gauss quadrature set is used.

\subsection{Simplified derivation for the CMFD Fourier analysis}

A simplified Fourier analysis starts with the definition of the Fourier ansatz as shown in Eq. (14). The tilde notation above a variable denotes the first order error term near the solution. The superscript $l$ and $l+1 / 2$ denote the CMFD iteration index and the 
intermediate transport iteration index between two consecutive CMFD solves. The subscript $k$ and $j$ represent the cell center (cell averaged) transport scalar flux and CMFD scalar flux. The $r$ in the transport scalar flux error term in Eq. (14) denotes the fine mesh index in each coarse mesh cell.

$$
\begin{aligned}
& \tilde{\Phi}_{j}^{(l+1 / 2)}=C^{(l+1 / 2)} e^{i \Sigma_{t} \lambda x_{j}} \\
& \tilde{J}_{j-1 / 2}^{n e t,(l+1 / 2)}=G^{(l+1 / 2)} e^{i \Sigma_{t} \lambda x_{j}} \\
& \tilde{\Phi}_{j}^{(l+1)}=R^{(l+1)} e^{i \Sigma_{t} \lambda x_{j}} \\
& \tilde{\phi}_{k}^{(l+1)}=E_{r}^{(l+1)} e^{i \Sigma_{t} \lambda x_{j}}
\end{aligned}
$$

As shown in Eq. (15), the higher order transport scalar flux in each coarse mesh cell is updated with the same ratio of the current iteration coarse mesh scalar flux over the $1 / 2$ iteration high order averaged coarse mesh scalar flux. The corresponding linearized error terms are then shown in Eq. (16), where the net effect of the second and third terms is the CMFD correction factor.

$$
\begin{gathered}
\phi_{k}^{(l+1)}=\phi_{k}^{(l+1 / 2)} \frac{\Phi_{j}^{(l+1)}}{\Phi_{j}^{(l+1 / 2)}}, k \in j, 1 \leq j \leq J \\
\tilde{\phi}_{k}^{(l+1)} \approx \tilde{\phi}_{k}^{(l+1 / 2)}+\tilde{\Phi}_{j}^{(l+1)}-\tilde{\Phi}_{j}^{(l+1 / 2)}
\end{gathered}
$$

With $q$ fine meshes in a coarse mesh cell, the transport scalar flux error term $E$ in each coarse mesh cell in Eq. (14) can be expressed in a vector form and the corresponding matrix form of Eq.(16) is shown in Eq. (17).

$$
\mathbf{E}^{(l+1)}=(\tilde{\mathbf{H}}+\mathbf{1}(\tilde{\mathbf{R}}-\tilde{\mathbf{C}})) \mathbf{E}^{(l)}, \mathbf{1} \equiv\left(\begin{array}{llll}
1 & 1 & \ldots & 1
\end{array}\right)^{T}
$$

where the $\tilde{\mathbf{H}}$ matrix is the error transition matrix for the transport calculation without CMFD acceleration and the CMFD correction term $\tilde{\mathbf{R}}-\tilde{\mathbf{C}}$ is defined in Eq. (18). The $\tilde{\mathbf{G}}$ vector in Eq. (18) is the error vector for the coarse mesh current. It is interesting to notice that the $\tilde{\mathbf{G}}$ and $\tilde{\mathbf{H}}$ have no direct dependence on the scattering. The detailed formulation for the $\tilde{\mathbf{G}}$ and $\tilde{\mathbf{H}}$ matrices can be found in [22] and is not included in this paper. 


$$
\tilde{\mathbf{R}}-\tilde{\mathbf{C}}=-\frac{\Delta\left(e^{i \Sigma_{t} \lambda \Delta}-1\right)}{D\left(2-2 \cos \left(\Sigma_{t} \lambda \Delta\right)\right)} \tilde{\mathbf{G}}
$$

As shown in Eq. (18), the CMFD correction term $\tilde{\mathbf{R}}-\tilde{\mathbf{C}}$ is inversely proportional to the diffusion coefficient and the new error transition matrix with the odCMFD method is shown in Eq. (19).

$$
\mathbf{E}^{(l+1)}=\left(\tilde{\mathbf{H}}+\frac{D}{D+\theta \Delta}(\tilde{\mathbf{R}}-\tilde{\mathbf{C}})\right) \mathbf{E}^{(l)}
$$

The error transition matrix defined in Eq. (18) and Eq. (19) are for a particular Fourier frequency, and the Fourier frequency is shown in Eq. (20) to satisfy the periodic boundary condition, where $X$ denotes the dimension of the $1 \mathrm{D}$ problem.

$$
\lambda=\frac{2 \pi s}{\Sigma_{t} X}, s=0,1,2,3 \ldots, J-1
$$

For each Fourier frequency and each $\theta$ value, the corresponding error reduction rate is expressed as the maximum eigenvalue of the error transition matrix.

$$
\omega(\lambda, \theta)=\max \left(\operatorname{abs}\left(\operatorname{eig}\left(\tilde{\mathbf{H}}+\frac{D}{D+\theta \Delta}(\tilde{\mathbf{R}}-\tilde{\mathbf{C}})\right)\right)\right)
$$

The spectral radius for a typical problem and a given $\theta$ value is thus the maximum error reduction rate over the whole Fourier frequency domain as shown in Eq. (22) and optimal value is determined by finding the minimum spectral radius for all $\theta$ value.

$$
\rho(\theta)=\max (\omega(\lambda, \theta))
$$

\subsection{Influence of the number of fine cells per coarse cell $q$ on the spectral radius}

In Figure 4, the spectral radius as a function of the value of $\theta$ for various values of $q$ (the number of fine cells per coarse cell) is investigated with three sets of optical thicknesses. The figure shows significantly different spectral radii for $q=1,2$ and 5 as a function of $\theta$ value. Also, the spectral radius saturates when $q$ is around 10. In realistic applications, the number of fine cells per coarse cell is usually about 10 , so $q$ is set to 10 for the rest of the analysis to be consistent with realistic applications. 

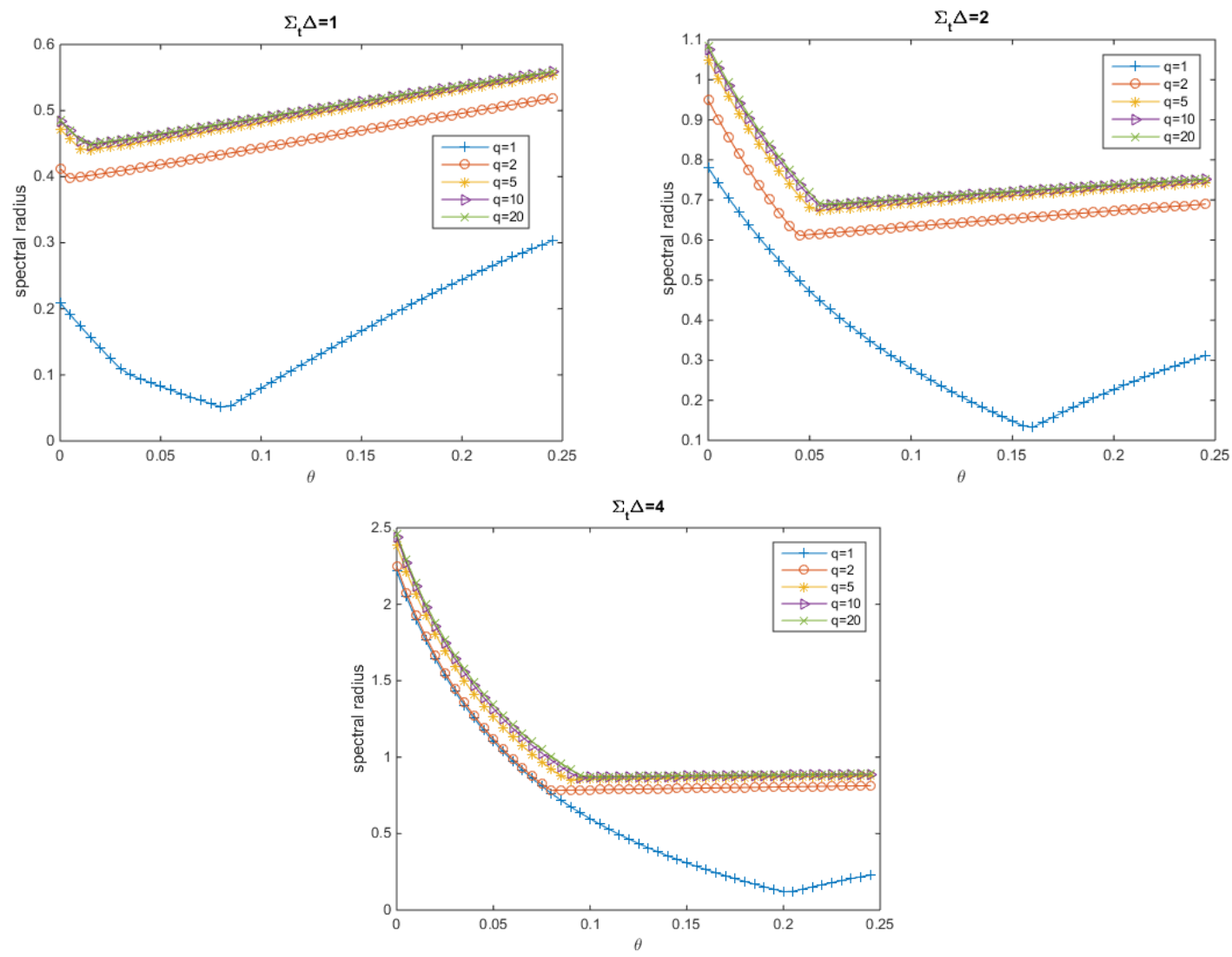

Figure 3: Effect of $q=$ number of fine cells per coarse cell.

\subsection{Spectral radius as a function of optical thickness}

The spectral radius as a function of $\theta$ for various coarse cell optical thicknesses is investigated in Figure 4. As illustrated, the optimal $\theta$ value increases as a function of optical thickness and is much smaller than the pCMFD value $1 / 4$. For a large optical thickness 4 , an optimal $\theta$ value around 0.1 considerably reduces the spectral radius to slightly above 0.8 , and the spectral radius increases slightly when the $\theta$ value continues increasing up to the $\theta$ used in the pCMFD method. For a small optical thickness 0.5 , the optimal $\theta$ is actually negative, and a minimal threshold of zero for the optimal $\theta$ is set because 1 ) the spectral radius with small optical thickness is already very small, and the impact of a negative $\theta$ value is not significant, and 2) a negative $\theta$ value may result in a negative unphysical diffusion coefficient and may cause an unnecessary stability problem. The spectral radius with optical thickness 0.5 increases slightly as a function of $\theta$ value above zero. 


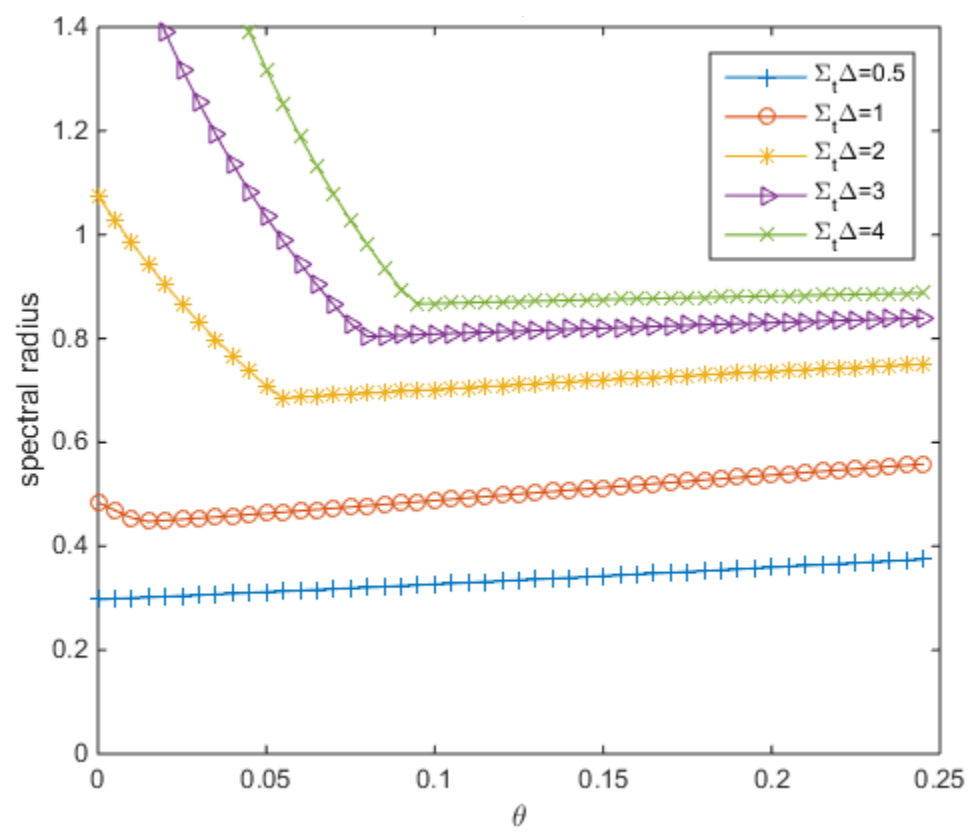

Figure 4: Spectral radius as a function of $\theta$ for various values of optical thickness

\subsection{Effect of the coarse mesh optical thickness on the spectral radius.}

If the optimal $\theta$ value is used for the artificially diffusive CMFD method, the spectral radius as a function of the optical thickness for CMFD, pCMFD and odCMFD are shown in Figure 5. As indicated, the CMFD spectral radius becomes greater than unity around an optical thickness value of 2, but the pCMFD is conditionally stable for any optical thickness. For an optical thickness smaller than 3, the CMFD method is faster than the pCMFD method. The newly proposed odCMFD method is as fast as the CMFD method for optical thickness smaller than 1 (no negative $\theta$ is allowed), is faster than both CMFD and pCMFD for optical thickness around 1 to 10 and is unconditionally stable for large optical thickness, where a very small reduction of spectral radius at large optical thickness is not noticeable for odCMFD compared to pCMFD. 


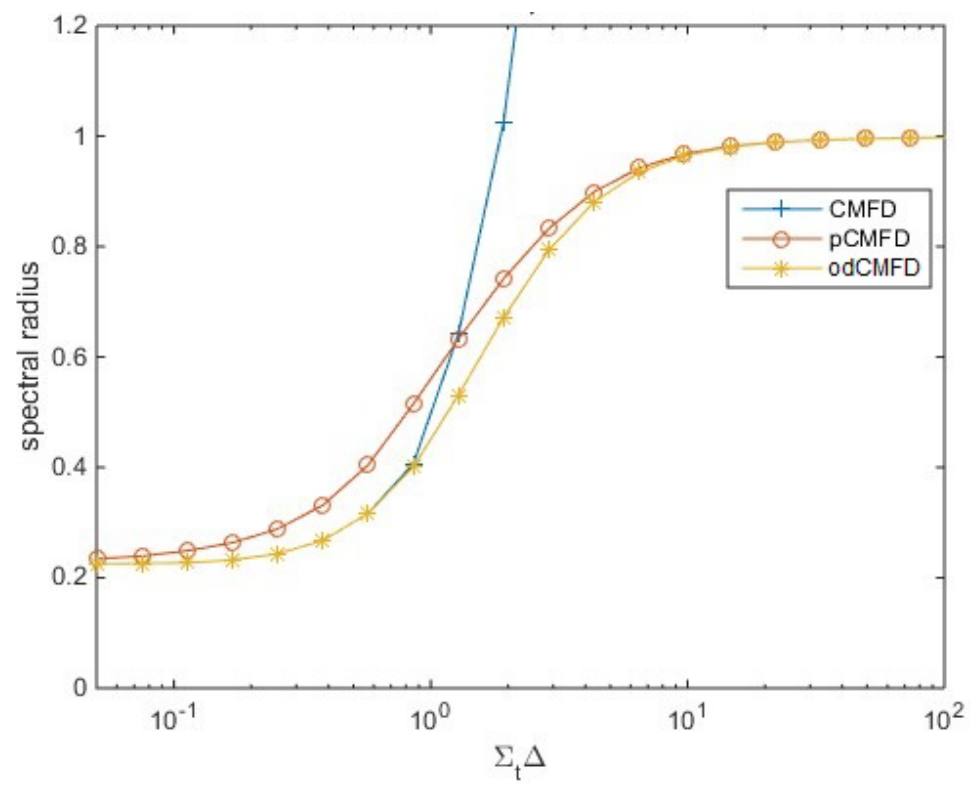

Figure 5: Comparison of three CMFD methods for various optical thickness

\subsection{Polynomial fitting for optimal spectral radius}

With the quadrature set and the $q$ values fixed, the only variable that impacts the spectral radius is the coarse cell optical thickness. As shown in [13] and in the Fourier analysis shown above, the scattering ratio has no impact on the spectral radius for one inner transport iteration per outer iteration, and the optimal $\theta$ value as a function of optical thickness is shown in Figure 6. As illustrated, the optimal $\theta$ is negative for optical thicknesses smaller than unity (no negative optimal $\theta$ is allowed), and saturates around 0.127 for optical thickness around 20. The saturated optimal $\theta$ is found to be much smaller than the pCMFD value $1 / 4$, indicating that the pCMFD method overcorrects the diffusion coefficient. 


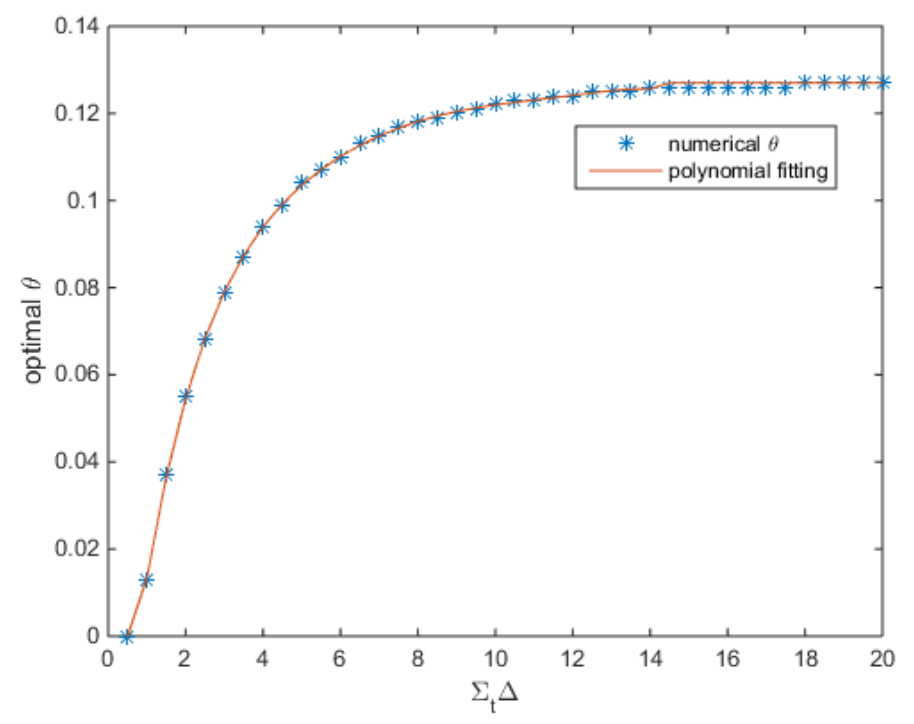

Figure 6: Numerical optimal $\theta$ and its polynomial fitting

A polynomial fitting based on Eq. (23) is also plotted in Figure 6, where the coefficients of the polynomial fitting are listed in Table 1.

$$
\theta=\left\{\begin{array}{cl}
0 & , \Sigma_{t} \Delta<1 \\
\sum_{i=0}^{6} a_{i}\left(\Sigma_{t} \Delta\right)^{i} & , 1 \leq \Sigma_{t} \Delta<14 \\
0.127 & , \Sigma_{t} \Delta \geq 14
\end{array}\right.
$$

Table 1 Coefficients for Polynomial Fitting of Optimal $\theta$ in Eq. (23)

\begin{tabular}{cc}
\hline Order $=i$ & Coefficient $=\boldsymbol{a}_{\boldsymbol{i}}$ \\
\hline 0 & $-5.542780 \mathrm{E}-02$ \\
1 & $8.740501 \mathrm{E}-02$ \\
2 & $-2.152599 \mathrm{E}-02$ \\
3 & $3.145553 \mathrm{E}-03$ \\
4 & $-2.683648 \mathrm{E}-04$ \\
5 & $1.222516 \mathrm{E}-05$ \\
6 & $-2.284879 \mathrm{E}-07$ \\
\hline
\end{tabular}




\section{NUMERICAL RESULTS}

In this section, the numerical results for CMFD, pCMFD and odCMFD are presented from applying the MPACT code to a homogeneous two region problem, a 2D C5G7 problem, the VERA benchmark problem 5, and a 2D BWR Peach Bottom Unit 2 core problem. The CMFD kernel in MPACT is used as the standard CMFD method and the $\theta$ value is set to be $1 / 4$ and the optimal polynomial fitted value for PCMFD and odCMFD respectively. The convergence criteria is set to $1 \mathrm{E}-6$ for all the problems in this section.

\subsection{Homogenized two-region problem}

In MPACT, the CMFD coarse mesh cell is defined as a pin cell, so the study of the coarse mesh size cannot be performed for single pin-cell problems. As a result, a two-region homogenized problem with realistic 56 energy group cross sections is used to investigate the difference of the three CMFD methods using various coarse mesh sizes. The detailed geometry of the homogeneous problem is shown in Figure 7, where the CMFD coarse mesh size varies from $0.5 \mathrm{~cm}$ to $8 \mathrm{~cm}$, and each coarse cell is subdivided into an array of $10 \times 10$ fine-mesh cells. A typical PWR pin material is homogenized as shown in Table 2 and the material in the upper left corner (red) is poisoned with boron while the rest (green) is free of boron. A ray spacing of $0.03 \mathrm{~cm}, 16$ azimuthal angles, and 3 polar angles with the Chebyshev-Yamamoto angular quadrature are used for all simulations.

Table 2: Homogenous two-region problem fuel composition

\begin{tabular}{cc}
\hline Isotope Name & Number density (barn-cm) \\
\hline U-234 & $2.03 \mathrm{E}-06$ \\
U-235 & $2.18 \mathrm{E}-04$ \\
U-236 & $1.10 \mathrm{E}-06$ \\
U-238 & $7.36 \mathrm{E}-03$ \\
H-1 & $3.31 \mathrm{E}-02$ \\
O-16 & $3.18 \mathrm{E}-02$ \\
B-10 & $1.80-06$ \\
B-11 & $2.88 \mathrm{E}-05$ \\
\hline
\end{tabular}




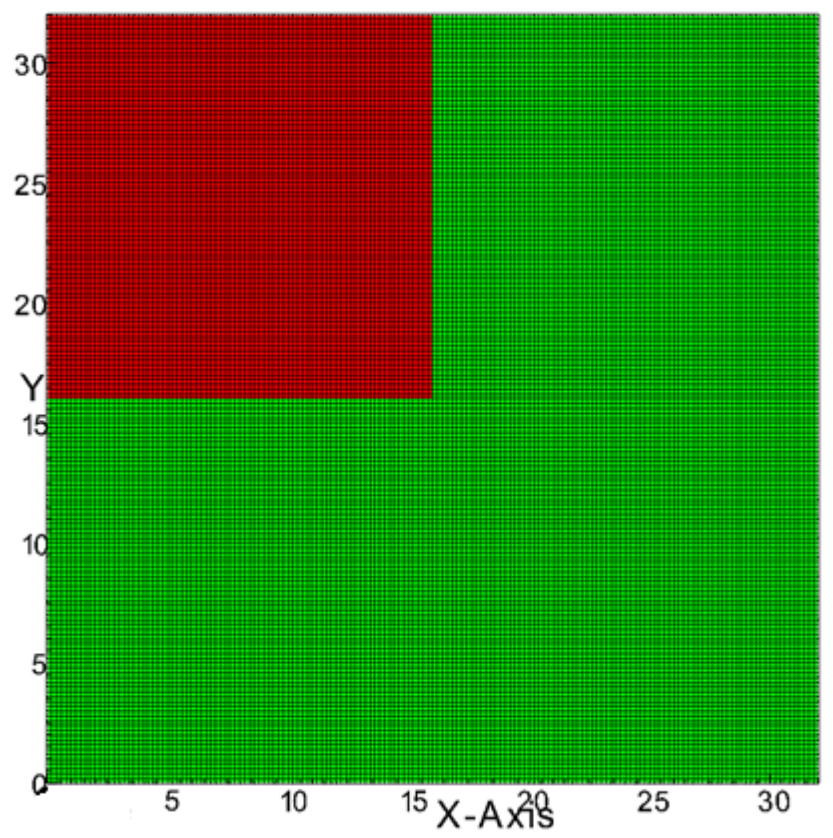

Figure 7: Homogenized two-region problem

Instead of presenting the spectral radius, the number of outers that truly accounts for the running time is tabulated in Table 3. As indicated, the odCMFD method is always faster than either the CMFD or the pCMFD methods. In more detail, with mesh size $8 \mathrm{~cm}$, CMFD fails to converge (as expected), while pCMFD and odCMFD are stable, with odCMFD faster than pCMFD by 6 outer iterations. With a mesh size of $4 \mathrm{~cm}$, CMFD still fails to converge and the odCMFD method is still faster by 3 outers than the pCMFD method. The case with a mesh size of $2 \mathrm{~cm}$ is the cross over point for the CMFD and pCMFD method, which is the same mesh size where CMFD starts to converge. As the mesh size continues to decrease, the PCMFD method loses its advantage over the CMFD method, while odCMFD is always as fast as the CMFD method. 
Table 3: Number of outers for homogenous two-region problem

\begin{tabular}{c|ccc}
\hline$\Delta(\mathbf{c m})$ & CMFD & PCMFD & odCMFD \\
\hline 0.5 & 3 & 5 & 3 \\
1 & 6 & 7 & 6 \\
2 & 16 & 11 & 9 \\
4 & Diverged & 23 & 20 \\
8 & Diverged & 57 & 51 \\
\hline
\end{tabular}

\subsection{D C5G7 results}

The 2D C5G7 [23] is a well-known benchmark problem that consists of a 17x17 fuel assembly with $\mathrm{UO}_{2}$ and MOX fuel pins. The assembly with $\mathrm{UO}_{2}$ fuel is used here to compare the efficiency of the three CMFD methods. Each pin of the C5G7 problem is modeled as a CMFD mesh, as shown in the left of Figure 8. A ray spacing of $0.03 \mathrm{~cm}, 16$ azimuthal angles, and 3 polar angles per octant with the Chebyshev-Yamamoto angular quadrature are used in this simulation.

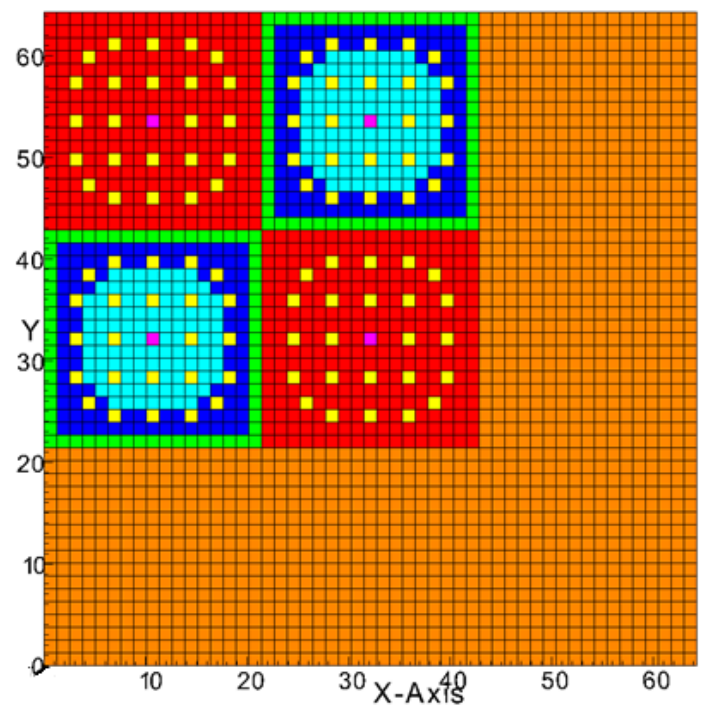

Figure 8 C5G7 CMFD cell layout

As noted previously, the default setting for MPACT models treats each fuel pin as a CMFD cell and therefore the coarse mesh size cannot be altered. The number of outer iterations for these three methods is shown in Table 4. As indicated, the odCMFD performs better than PCMFD and the CMFD fails to converge. 


\section{Table 4: Number of outers for C5G7 2D problem}

\begin{tabular}{ccc}
\hline CMFD & PCMFD & odCMFD \\
\hline Diverged & 19 & 18 \\
\hline
\end{tabular}

\subsection{VERA Problem 5}

The VERA Benchmark 5 problem provides a realistic full core model based on the Watts Bar PWR. It is one of a sequence of problems from the CASL (Consortium for Advanced Simulation of LWRs) VERA (Virtual Environment for Reactor Applications) benchmark progression [24]. The problem is a 2D slice of the Watts Bar startup core, with Westinghouse $17 \times 17$-type fuel assemblies at beginning-of-life and hot zero power isothermal conditions. The assembly loading pattern for this problem is shown in Figure 9. A ray spacing of $0.05 \mathrm{~cm}, 4$ azimuthal angles, and 4 polar angles per octant with the Chebyshev-Yamamoto angular quadrature are used in this simulation.

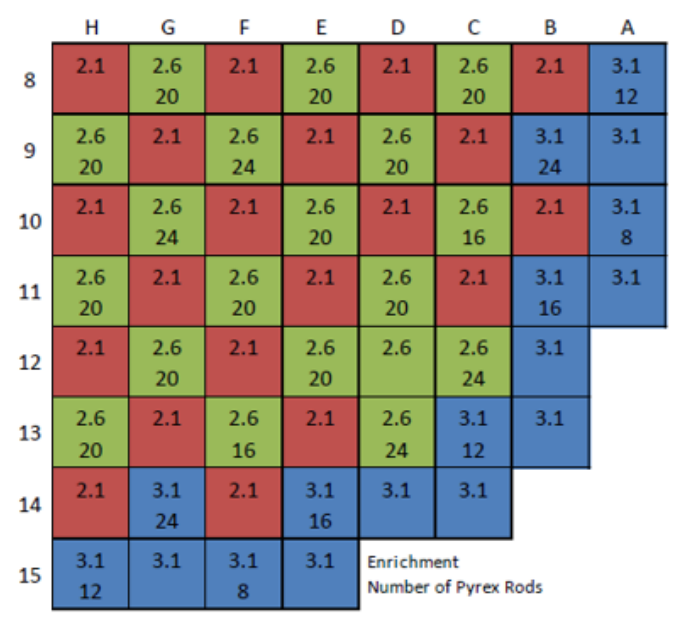

Figure 9 Problem 5 assembly and poison loading pattern [24]

The number of outer iterations for these three CMFD methods are documented in Table 5. As indicated, the VERA problem is not a difficult problem for standard CMFD and it converges in 15 iterations. The PCMFD is one iteration slower than the CMFD and the odCMFD is the fastest among all three methods, which is one iteration faster than CMFD and two iterations faster than PCMFD.

Table 5: Number of outers for VERA Problem 5

\begin{tabular}{ccc}
\hline CMFD & pCMFD & odCMFD \\
\hline 15 & 16 & 14 \\
\hline
\end{tabular}




\subsection{Full Core Peach Bottom Unit 2 Beginning of Cycle 1 (2-D)}

The final problem is for a BWR and is taken from the Peach Bottom Unit 2 beginning of cycle 1 [25]. A 2D full core model was used as shown in Figure 10 with a MOC discretization of $0.05 \mathrm{~cm}$ ray spacing and 8 azimuthal and 2 polar angles per octant with the Chebyshev-Yamamoto angular quadrature. Each pin of the Peach Bottom problem is modeled as a CMFD mesh.

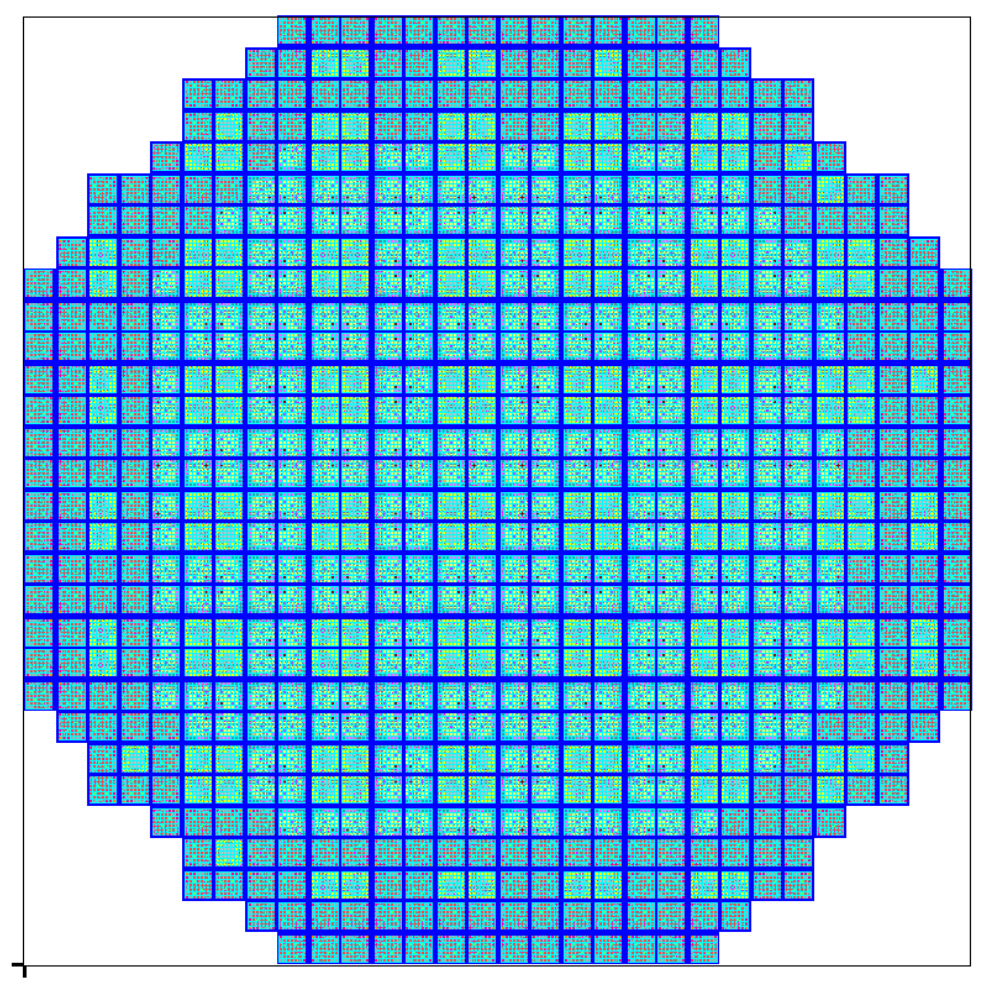

Figure 10 Full Core Peach Bottom Unit 2 Beginning of Cycle 1 (2-D)

The number of outer iterations for these three CMFD methods are shown in Table 6. The CMFD fails to converge for the 2D Peach Bottom problem while the pCMFD and odCMFD are both stable. The odCMFD is the most efficient among these three methods and is 3 iterations faster than pCMFD.

Table 6: Number of outers for 2D BWR Peach Bottom Unit 2 Core Problem

\begin{tabular}{ccc}
\hline CMFD & PCMFD & odCMFD \\
\hline Diverged & 33 & 30 \\
\hline
\end{tabular}




\section{SUMMARY AND CONCLUSIONS}

In this paper, an optimally diffusive CMFD (odCMFD) method is proposed, based on the CMFD and pCMFD method by adding an artificial term to the standard diffusion coefficient. The traditional CMFD method and PCMFD methods preserve the net current and partial currents of the high order solution respectively, and essentially differ in the low-order diffusion coefficient only by an additive "artificially diffusive" parameter of one quarter times the coarse mesh thickness. Numerical experiments and theoretical analyses confirm previous study results and show that CMFD is faster than PCMFD for thin coarse meshes, but diverges for large coarse meshes, however pCMFD is unconditionally stable for all coarse mesh thicknesses.

The artificially diffusive term in the odCMFD method was investigated using a Fourier analysis based on a monoenergetic infinite homogenous-medium problem for various coarse cell optical thicknesses and scattering ratios. The results predict that the optimal $\theta$ increases as the optical thickness increases, and has no dependence on the scattering ratio. The optimal $\theta$ is always smaller than the pCMFD predicted value $1 / 4$, which indicates that pCMFD overcorrects the diffusion coefficient and thus unnecessarily increases the spectral radius. A simple polynomial fitting was developed for implementation in the MPACT code to perform calculations for realistic problems. This fitting can be easily implemented in other transport/CMFD based neutron simulation codes.

Numerical results from MPACT for a two-region homogeneous problem, a 2D C5G7 problem, a 3-D PWR quarter core and a 2D BWR Peach Bottom Unit 2 core problem agree well with the Fourier analysis predicting the behavior of the CMFD, PCMFD and odCMFD methods. These results show: 1) CMFD converges faster than PCMFD for optically thin coarse mesh cells but diverges for optically thick coarse mesh cells; 2) pCMFD is unconditionally stable for all coarse mesh cells; and 3) the odCMFD method is always the most efficient among these three methods. The odCMFD method has a similar convergence rate as CMFD for optically thin coarse mesh cells, is faster than both CMFD and pCMFD for coarse mesh cells with intermediate optical thickness, and is unconditionally stable and faster than PCMFD for optically thick coarse mesh cells.

In conclusion, the odCMFD method should converge at least as fast as CMFD or faster and should always converge faster than pCMFD method without ever becoming unstable. To implement odCMFD, only a minor coding effort should be required to modify an existing standard CMFD (or pCMFD) implementation. The effort to modify CMFD to odCMFD should also be significantly less than that required to modify CMFD to pCMFD. 
Ongoing work has included a detailed study about the odCMFD method compared to the theoretical CMFD convergence rate lower bounds. Results indicate that the odCMFD is essentially very close to the theoretical CMFD convergence rate lower bounds.

\section{ACKNOWLEDGMENTS}

The authors would like to thank Kendra Keady and Blake Kelly for their help with the Fourier analysis and for discussions about the artificially diffusive idea. This research was supported by the Consortium for Advanced Simulation of Light Water Reactors (www.casl.gov), an Energy Innovation Hub (http://www.energy.gov/hubs) for Modeling and Simulation of Nuclear Reactors under U.S. Department of Energy Contract No. DEAC05-00OR22725. 


\section{REFERENCES}

[1] Kochunas, B., et al., "VERA Core Simulator Methodology for PWR Cycle Depletion." Nuclear Science and Engineering, submitted.

[2] Zhu, A., et al., "A Multi-level Quasi-Static Kinetics Method for Pin-Resolved Transport Transient Reactor Analysis”, Nuclear Science and Engineering, 2016, 182(4).

[3] Zhu, A., et al., "Stability analysis of the Backward Euler time discretization for the pin-resolved transport transient reactor", Annals of Nuclear Energy, 2016, 87(2):252-266.

[4] Adams, M., and Larsen, E., "Fast Iterative Methods for Discrete Ordinates Parti cle Transport Calculations," Progress in Nuclear Energy, 40, 3 (2002).

[5] Li, L., "A low order acceleration scheme for solving the neutron transport equation”, Master Dissertation, Massachusetts Institute of Technology, 2013.

[6] Smith, K., "Nodal Method Storage Reduction by Nonlinear Iteration," Trans. Am. Nucl. Soc., 44, 265, (1983).

[7] Downar, T., Xu, Y., and Seker V., "PARCS v3.0 U.S. NRC Core Neutronics Simulator User/Theory Manual”, Department of Nuclear Engineering and Radiological Sciences, University of Michigan, Ann Arbor, MI, (2009).

[8] MPACT Team, "MPACT Theory Manual, Version 1.0", University of Michigan, Ann Arbor, MI, October, (2013).

[9] Zhu, A., et al., "Transient Methods For Pin-Resolved Whole Core Transport Using the 2D-1D Methodology in MPACT", Proc. M\&C 2015, American Nuclear Society, Nashville, TN, USA, April 19-23, (2015).

[10] Cho, J. et al., "Transient Capability for a MOC-Based Whole Core Transport Code DeCART,” Trans. Am. Nucl. Soc., 92, 721 (2005).

[11] SHANER, S., FORGET, B., and SMITH, K., "Sensitivity Analysis and Performance of the Adiabatic, Theta, and Multigrid Amplitude Function Kinetics Methods in 2D MOC Neutron Transport," Proc. M\&C 2013, Sun Valley, ID, May 5-9, (2013).

[12] Jarrett, M., et al, "Stabilization Methods for CMFD Acceleration," Proc. M\&C 2015, American Nuclear Society, Nashville, TN, USA, April 19-23, (2015) 
[13] Keady, K., and Larsen, E., "Stability of SN K-Eigenvalue Iterations Using CMFD Acceleration,” Proc. M\&C 2015, American Nuclear Society, Nashville, TN, USA, April 19-23, (2015).

[14] Lee, D., Downar, T., and Kim, Y., "Convergence Analysis of the Nonlinear Coarse-Mesh Finite Difference Method for One-Dimensional Fixed-Source Neutron Diffusion Problem,” Nuclear science and engineering, 147, 127 (2004).

[15] Hong, S., Kim, K., and Song, J., "Fourier Convergence Analysis of the Rebalance Methods for Discrete Ordinates Transport Equations in Eigenvalue Problems,” Nuclear Science and Engineering, 164, 33 (2010).

[16] Cho, N., et al., "Partial Current-Based CMFD Acceleration of the 2D/1D Fusion Method for 3D Whole-Core Transport Calculations", Trans. Am. Nucl. Soc., 88, 594 (2003).

[17] Cho, N., "Partial Current-Based CMFD (p-CMFD) Method Revisited”, Trans. Korea Nucl. Soc., Autumn Meeting, Gyeongju, Korea, Oct. 25-26 (2012).

[18] Jarrett, M., "Analysis of Stabilization Techniques for CMFD Acceleration of Neutron Transport Problems," Nuclear Science and Engineering, Submitted.

[19] Larsen, E., "Infinite Medium Solutions of the Transport Equation, $\mathrm{S}_{\mathrm{N}}$ Discretization Schemes, and the Diffusion Approximation,” Transport Theory and Statistical Physics, 32, 633 (2003).

[20] Yamamoto, A., "Generalized Coarse-Mesh Rebalance Method for Acceleration of Neutron Transport Calculations," Nuclear Science and Engineering, 151(3), 274-282 (2005).

[21] Kelley, B., and Larsen, E., "A consistent 2D/1D approximation to the 3D neutron transport equation,” Nuclear Engineering and Design, 295 (2015): 598614.

[22] Zhu, A., et al, "Theoretical Convergence Rate Lower Bounds for Variants of Coarse Mesh Finite Difference to Accelerate the Neutron Transport Calculations," Nuclear Science and Engineering, In Preparation.

[23] Lewis, E., et al, "Benchmark specification for Deterministic 2-D/3-D MOX fuel assembly transport calculations without spatial homogenization (C5G7 MOX),” NEA/NSC (2001).

[24] Godfrey, A., "VERA core physics benchmark progression problem specifications," Consortium for Advanced Simulation of LWRs. Oak Ridge, 
TN: Oak Ridge National Laboratory (2013).

[25] Larsen, N., "Core Design and Operating Data for Cycles 1 and 2 of Peach Bottom 2,” EPRI Technical Report, NP-563, June, (1978). 